Original Article

\title{
Specificity and sensitivity as important parameters of the pattern of the homeopathic remedy
}

\author{
M. Ashikhmina \\ System Homeopathy Center, Moscow. E-mail: ma@syshom.ru
}

\begin{abstract}
Aim: To assess the opportunity of use specificity ( $\mathrm{Sp}$ ) and sensitivity (Se) - indicators that are generally accepted for any diagnostic method - in homeopathy.

Methodology: Analysis of comprehensions Specificity and Sensitivity in context of homeopathic knowledge.

Results and discussions: In homeopathy, as in mathematics, patterns are identified through research, the main resources: provings, cases, repertory data, Materia Medica, and source information. S. Bohger wrote: "not all symptoms are equivalent", "peculiar characteristics should be distinguished from the general series". Constdering the different facets in the general pattern of any remedy, we can see that the weight and diagnostic significance of each facet are different. Each facet of the pattern can be characterized by specificity (Sp) and sensitivity (Se) - indicators that are generally accepted for any diagnostic method. Specificity in medicine is the ability of the diagnostic method (facet of the pattern) not to give false positive results in the absence of disease (i.e., not to point to another remedy). Sp shows to what degree this parameter is typical for this remedy. It is defined as the proportion of the truly negative results of healthy individuals in the group under examination (i.e., the more often the fafet s fould in they remedies, the lower the specificity is). E.g., the Sp of sore throat for Hepar sulphtrlis howrely the sore throat will be met in all other cases, except Hepar sulphur). Sensitivity is the ability of the diagnostic method (certain facet of the pattern) to give the correct result (to identify the remedy), which is defined as the proportion of true positive results among all tests performed (i.e., how often this facet of the pattern appears in this remedy compared to others). The Se reflects how often this pattern theme (symptom) occurs among all cases of this remedy, for example, how often we will find the sore throat among all cases of Hepar sulphur. The key symptoms, corresponding to the leading symptoms of the remedy, socalled "grains of gold" in homeopathy, can be attributed to highly specific parameters: they "valuable, few and hidden", and as the "key to the lock" opening the whole case. Obviously, the key symptoms should have both high Sp and sufficient Se to be some kind of a visiting card of the remedy. Concomitant symptoms can be attributed to sensitive parameters, they complement the overall picture of the remedy, but they cannot be relied on in the decisive choice of the remedy (due to their low specificity).
\end{abstract}


International Journal of High Dilution Research 2018; 17(2):31-32

Available online at www.highdilution.org

Conclusion: Sp and Se can use for description basic homeopathic terms and concepts and show diagnostic contribution of individual parameters in total pattern of the homeopathic remedy on different levels of disease.

Received: March 1, 2018. Accepted: April 26, 2108.

(C) International Journal of High Dilution Research.

Not for commercial purposes. 